\title{
Nucleic Acid Sensing onto Peptide Nucleic Acid (PNA) Modified Solid Surfaces
}

\section{Ghosh S and Mukhopadhyay $\mathbf{R}^{*}$}

Department of Biological Chemistry, Indian Association for the Cultivation of Science, India

A biosensor, which is conventionally defined as "a device that uses specific biochemical reactions mediated by isolated enzymes, immunosystems, tissues, organelles or whole cells, to detect chemical compounds usually by electrical, thermal or optical signals" [1], comprises of at least two basic steps: first, target recognition and second, signal transduction. In nucleic acid sensing, the single-stranded DNA/ RNA probes are often employed as the sensor probes, since by virtue of their capacity of binding to their complementary target nucleic acid strands with high sequence-specificity, they can almost always detect the presence of the specific target strands in the sample analyte solution, without error. Regarding transduction of the molecular recognition event into an electronic signal, mostly the optical, electrochemical, electrical, mechanical, acoustic or thermal methods are applied in the current nucleic acid sensors [2-6].

Immobilization of the nucleic acid sensor probes onto a solid substrate in the surface-based nucleic acid sensors is one of the crucial initial steps that can play a decisive role in the overall performance of the sensor. Ideally, the nucleic acid strands are to be immobilized onto the solid support in such a way that a specific recognition signal can be obtained only if they recognize their target probes via sequence-specific hybridization interactions. The experimental setup should be such that the various non-specific interactions, e.g. nucleobase-substrate interactions as in case of interactions between the nucleobase nitrogen and a gold substrate, are largely cancelled out or do not take place to a significant extent. Hence, experimental conditions must be adjusted for every application, and a large choice of immobilization support and methods should be considered before the most optimal arrangement can be identified. The immobilization strategy could include primarily two means, one, covalent binding via one end of the nucleic acid probe, e.g. binding of 5'-thiol-modified DNA oligonucleotides onto gold surface via gold-sulfur interactions [7], or binding of a $5^{\prime}$-aminomodified DNA oligonucleotide onto an epoxy-modified surface [8], and two, non-covalent binding (e.g. affinity binding based on the strong avidin biotin system, where nucleic acid is biotinylated at its 5 ' end and the avidin is attached directly to carbon-based surface [9].

While quite high sensitivity (femto molar to atto molar range) in target detection has been exemplified in optical/electrochemical transduction $[6,10]$, another approach for sensitivity enhancement, i.e. by application of synthetic nucleic acid analogues like peptide nucleic acid (PNA) [11] and locked nucleic acid (LNA) [12,13] probes, which are capable of forming more stable duplexes with the DNA targets than the DNA sensor probes, may also be explored. The unique physicochemical nature of the peptidic, non-ionic backbone of PNA has promoted the use of PNA oligomers as capture probes in electrochemical, optoelectronic sensors, and microarray-based sensors $[3,14,15]$.

PNA is an artificially synthesized polymer, invented by Peter E. Nielsen (University of Copenhagen), Michael Egholm (University of Copenhagen), Rolf H. Berg (Risø National Lab) and Ole Buchardt (University of Copenhagen) during the 1990s [11]. PNA is a DNA analog, in which a 2-aminoethyl-glycine linkage generally replaces the normal phosphodiester backbone [11,16]. A methyl carbonyl linker connects natural, as well as unusual (in some cases), nucleotide bases to this backbone at the amino nitrogens. PNA is non-ionic, achiral, and is not susceptible to hydrolytic (enzymatic) cleavage. PNAs are capable of sequence-specific binding with complementary DNA, as well as RNA obeying the Watson-Crick base paring $[17,18]$. Its hybrid complexes exhibit extraordinary thermal stability. PNA has high affinity to its complementary DNA or RNA molecules, mainly because of the lack of electrostatic repulsion between the uncharged PNA backbone, and that of the natural nucleic acid. Within the PNA hybrids, the order of the thermal stability of the duplexes is found to be PNA-PNA $>$ PNARNA $>$ PNA-DNA [19].

In contrast to the DNA-DNA duplexes, the stability of the PNA-DNA hybrids is not significantly affected by changes in ionic strength, except in the limit of low ionic strength, i.e. when the salt concentration is few $\mathrm{mM}$, where the stability increases. The binding of PNA to a corresponding complementary DNA oligomer takes place in a sequence-specific manner, which means that the thermal stability of a hetero-duplex, where PNA is one of the components, can be considerably lowered by the presence of imperfect matches. Owing to the high sequence specificity of PNA binding to other nucleic acid strands, incorporation of any mismatch in the duplex considerably affects the $\mathrm{T}_{\mathrm{m}}$ value of the hetero-duplex. For example, a single base mismatch could result in the lowering of the $T_{m}$ value by $15^{\circ} \mathrm{C}$ and $11^{\circ} \mathrm{C}$, in case of the 15 mer PNA-DNA and DNA-DNA duplexes, respectively [20]. This property of PNA is responsible for the remarkable discrimination between perfect matches and mismatches offered by PNA probes, and makes PNA attractive as oligonucleotide recognition elements in biosensor technologies [20].

Application of PNA as a sensor probe in nucleic acid sensor technologies holds great promise for rapid (since formation of PNA-DNA duplex is faster than formation of DNA-DNA duplex) and cost-effective detection (since lesser amount of sample is needed due to high sensitivity in PNA-based measurements) of specific DNA sequences. Usually, single-stranded PNA (ssPNA) probes are immobilized onto the transducer surface by chemical means, e.g. gold-sulfur bond formation for immobilization of thiolated ssPNA probes onto the gold surface [20]. Once the PNA sensor probes can detect the complementary (or non-complementary) target nucleic acid

*Corresponding author: Mukhopadhyay R, Department of Biological Chemistry, Indian Association for the Cultivation of Science, Jadavpur, Kolkata-700 032, India, Tel: 91-33-2473-4971; Fax. 91-33-2473-2805; E-mail: bcrm@iacs.res.in

Received September 19, 2013; Accepted September 22, 2013; Published September 25, 2013

Citation: Ghosh S, Mukhopadhyay R (2013) Nucleic Acid Sensing onto Peptide Nucleic Acid (PNA) Modified Solid Surfaces. J Bioanal Biomed 5: e120. doi:10.4172/1948-593X.1000e120

Copyright: ( 2013 Ghosh S, et al. This is an open-access article distributed under the terms of the Creative Commons Attribution License, which permits unrestricted use, distribution, and reproduction in any medium, provided the original author and source are credited. 
strands in the sample solution, the response from the hybridization event or a lack of it is converted into a useful electronic response by the transducer. The first report by Jensen et al. [19] in 1997 on detection of PNA-DNA and PNA-RNA hybridization using surface plasmon resonance (SPR), showed that the sensor can differentiate between a complementary and a non-complementary oligonucleotide sequence. The sensor chip used in this case was a thin gold film covered with a layer of dextran and containing streptavidin, chemically coupled to the dextran layer. Biotinylated PNA molecules were immobilized on the surface by means of strong coupling between biotin and streptavidin. The amount of the bound substance (complementary, as well as various non-complementary DNA and RNA oligonucleotides) was measured as a function of time, when a solution containing the target strands was flown over the chip surface. In this way, the association kinetics could be studied. The quartz crystal microbalance (QCM) mass measuring method can also be applied in studying hybridization of nucleic acids on solid surfaces [21-23]. The first report about the study of PNADNA hybridization using the QCM biosensor and PNA as the sensor probes comes from the work of Wang et al. [24], which showed that the system could differentiate between complementary and noncomplementary oligonucleotides. A fast and sensitive detection of mismatched sequences was made possible by monitoring the frequency $v s$. time response of the PNA-based QCM sensor. The PNA molecules used in the above-mentioned study contained a cysteine attached to the PNA strand with the help of an ethylene glycol unit, and a PNA monolayer could be formed onto the gold-coated quartz crystal surface using this thiol-PNA construct [24]. The immobilized PNA probes exhibited remarkable sequence specificity and gave rise to rapid hybridization with the target oligonucleotides sequences. The use of PNA as recognition probe for detection of target nucleic acid strand using electrochemical means has been reported, as early as in 1998, by Wang [20]. Their method consisted of four steps: PNA probe immobilization onto the transducer surface (here, a carbon paste electrode), hybridization to target DNA strands, indicator binding and chronopotentiometric transduction. The hybridization experiment was carried out by immersing the electrode into the stirred buffer solution containing a desired target, followed by measurement of signal.

Motivated by the previous reports that PNA can potentially be a better alternative for on-surface nucleic acid detection technologies compared to DNA probes, the Mukhopadhyay group developed a sensitive and robust bio-active self assembled PNA sensor layer, which is capable of efficient and specific target detection. The formation of close association of ssPNA strands was found to be relatively straightforward, and compact self-assembled PNA films could be readily generated on solid substrates like gold (111) surface by a simple immersion method [25]. In such films, the immobilized ssPNA strands could be oriented away from the surface, as elicited from reflection absorption infrared spectroscopy (RAIRS) experiments [25], and nonspecific interactions with the underlying gold substrate could be largely avoided, creating an ideal situation for the target nucleic acid strands to access the immobilized sensor PNA probes. On the contrary, the DNA films comprising the negatively charged ssDNA strands have been found to be mostly disordered/poorly ordered [26], where nonspecific DNA-surface interactions could occur through the relatively exposed nucleobases, resulting in reduced bioactivity of the film [27]. Ghosh et al. [28] have recently reported that the mismatch discrimination ability of such surface-anchored PNA layers could be successfully enhanced via ionic control, i.e. by varying salt concentration and the type of counterion. While the nature of ionic dependence of 'on-surface' behavior of PNA probes deviated significantly from the 'solution' behavior of these probes, e.g. in case of the singly mismatched duplexes, considerable similarities were also observed, e.g. in case of the fully mismatched duplexes. The single base mismatch discrimination capacity of the PNA probes could be further amplified by controlling PNA probe density using a nanoparticle-based approach, where gold (111) surface was modified with gold nanoparticles prior to PNA adsorption [29]. The simple strategy for formation of the surface-attached AuNP-PNA construct appeared to be beneficial not only because the difficulty in attaching PNA probes onto AuNPs, without AuNPs getting aggregated, could be overcome, but importantly, because this allowed an increase in the sensor probe density, and therefore, increase in the hybridization probability. Considering the need for developing sensitive, targetspecific and robust high-throughput array technologies, PNA-based nucleic acid detection assays, as presented in this report, could offer practical inputs in achieving better control on on-surface DNA detection capabilities.

\section{References}

1. Newman JD, Tigwell LJ, Turner APF, Warner PJ (2004) Biosensors: A clearer view. Cranfield University, UK.

2. Ermini ML, Mariani S, Scarano S, Campa D, Barale R, et al. (2013) Single nucleotide polymorphism detection by optical DNA-based sensing coupled with whole genomic amplification. Anal Bioanal Chem 405: 985-993.

3. Wang J, Palecek E, Nielsen PE, Rivas G, Cai X, et al. (1996) Peptide nucleic acid probes for sequence-specific DNA biosensors. J Am Chem Soc 118 7667-7670.

4. Fritz J, Baller MK, Lang HP, Rothuizen H, Vettiger P, et al. (2000) Translating biomolecular recognition into nanomechanics. Science 288: 316-318.

5. Fourati N, Lazerges M, Vedrine C, Fougnion JM, Zerrouki C et al. (2009) Surface acoustic waves sensor for DNA-biosensor development. Sensor Lett 7: $1-4$.

6. Ask J, Mukhopadhyay R, Hansen JO, Gothelf K (2006) Femtomolar electrochemical detection of DNA-targets using metalsulfide nanoparticles. J Am Chem Soc 128: 3860-3861.

7. Nuzzo RG, Allara DL (1983) Adsorption of bifunctional organic disulfides on gold surfaces. J Am Chem Soc 105: 4481-4483.

8. Lamture JB, Beattie KL, Burke BE, Eggers MD, Ehrlich DJ, et al. (1994) Direct detection of nucleic acid hybridization on the surface of a charge coupled device. Nucleic Acids Res 22: 2121-2125

9. Labuda J, Brett AMO, Evtugyn G, Fojta M, Mascini M, et al., (2010) Electrochemical nucleic acid-based biosensors: concepts, terms, and methodology. J Wang Pure Appl Chem 82: 1161-1187.

10. Liu YQ, Zhang M, Yin BC, Ye BC (2012) Attomolar ultrasensitive microRNA detection by DNA-scaffolded silver-nanocluster probe based on isothermal amplification. Anal Chem 84: 5165-5169.

11. Nielsen PE, Egholm M, Berg RH, Buchardt O (1991) Sequence selective recognition of DNA by strand displacement with a thymine substituted polyamide. Science 254: 1497-1500.

12. Singh SK, Nielsen P, Koshkin AA, Wengel J (1998) LNA (locked nucleic acids): synthesis and high-affinity nucleic acid recognition. Chem Commun 455-456.

13. Koshkin AA, Nielsen P, Meldgaard M, Rajwanshi VK, Singh SK, et al., (1998) LNA (Locked Nucleic Acid): An RNA mimic forming exceedingly stable LNA: LNA duplexes. J Am Chem Soc 120: 13252-13253.

14. Endo T, Kerman K, Nagatani N, Takamura Y, Tamiya E (2005) Label-free detection of peptide nucleic acid-DNA hybridization using localized surface plasmon resonance based optical biosensor. Anal Chem 77: 6976-6984

15. Okahata Y, Matsunobo Y, ljiro K, Mukae M, Murakami A, et al. (1992) Hybridization of nucleic-acids immobilized on a quartz crystal microbalance. $J$ Am Chem Soc 114: 8299-8300.

16. Egholm M, Buchardt O, Nielsen PE, Berg RH (1992) Peptide nucleic acids (PNA) - oligonucleotide analogues with an achiral peptide backbone. J Am Chem Soc 114: 1895-1897. 
Citation: Ghosh S, Mukhopadhyay R (2013) Nucleic Acid Sensing onto Peptide Nucleic Acid (PNA) Modified Solid Surfaces. J Bioanal Biomed 5: e120. doi:10.4172/1948-593X.1000e120

17. Egholm M, Buchardt O, Christensen L, Behrens C, Frier SM, et al. (1993) PNA hybridizes to complementary oligonucleotides obeying the Watson-Crick hydrogen bonding rules. Nature 365: 566-568

18. Egholm M, Nielsen PE, Buchardt O, Berg RH (1992) Recognition of guanine and adenine in DNA by thymine and cytosine containing peptide nucleic acids. J Am Chem Soc 114: 9677-9678.

19. Jensen KK, Orum H, Nielsen PE, Norden B (1997) Kinetics for hybridization of peptide nucleic acids (PNA) with DNA and RNA studied with the BIAcore technique. Biochemistry 36: 5072-5077.

20. Wang J (1998) DNA biosensors based on Peptide Nucleic Acid (PNA) recognition layers. Biosens Bioelectron 13: 757-762.

21. Okahata Y, Matsunobo Y, ljiro K, Mukae M, Murakami A, et al. (1992) Hybridization of nucleic-acids immobilized on a quartz crystal microbalance. Am Chem Soc 114: 8299-8300.

22. Okahata Y, Niikura K, Sugiura Y, Sawada M, Morii T (1998) Kinetic studies of sequence-specific binding of GCN4- bZIP peptides to DNA strands immobilized on a $27-\mathrm{MHz}$ quartz-crystal microbalance. Biochemistry $37: 5666-5672$.

23. Lina L, Zhaoa H, Lia J, Tanga J, Duanb M, et al. (2000) Study on Colloidal
Au-Enhanced DNA Sensing by Quartz Crystal Microbalance. Jiang L Biochem. Biophys Res Commun 274: 817-820.

24. Wang J, Nielsen PE, Jiang M, Cai X, Fernandes JR, et al., (1997) Mismatchsensitive hybridization detection by peptide nucleic acids immobilized on a quartz-crystal microbalance. Anal Chem 69: 5200-5202.

25. Ghosh S, Mukhopadhyay R (2011) An atomic force microscopy investigation on self-assembled peptide nucleic acid structures on gold(111) surface. Colloid Interface Sci 360: 52-60.

26. Wang H, Tang Z, Li Z, Wang E (2001) Self-assembled monolayer of ssDNA on $\mathrm{Au}(111)$ substrate. Surf Sci 480: L389-L394.

27. Herne TM, Tarlov MJ (1997) Characterization of DNA probes immobilized on gold surfaces. J Am Chem Soc 119: 8916-8920.

28. Ghosh S, Mishra S, Banerjee T, Mukhopadhyay R (2013) Facilitating mismatch discrimination by surface-affixed PNA probes via ionic regulation. Langmuir 29: 3370-3379.

29. Enhancing on-surface mismatch discrimination capability of PNA probes by AuNP modification of gold(111) surface. Ghosh S, Mishra S, Mukhopadhyay R (2013) Langmuir. 